\title{
Prevenzione delle infezioni catetere venoso centrale correlate (CRBSI)
}

\author{
Marina Cornacchiari' ${ }^{1}$, Maurizio Gallieni' ${ }^{2}$, Antonella Stasi ${ }^{1}$, Maria Giuseppina Ponticelli, \\ Barbara Gidaro ${ }^{1}$, Carlo Guastoni ${ }^{1}$
}

\author{
${ }^{1}$ Azienda Ospedaliera Legnano, Presidio Magenta, reparto di Nefrologia e Dialisi, Magenta (MI) \\ ${ }^{2}$ Ospedale San Carlo Borromeo, Direttore reparto di Nefrologia e Dialisi, Milano
}

\begin{abstract}
Prevention of Catheter-Related blood STREam infections (CRBSI)
Abstract. The use of temporary or tunneled central venous catheters $(\mathrm{CVC})$ is increasing worldwide in hemodialysis patients, but their numerous and potentially severe complications should be taken into account. Among the possible complications, infections are certainly those with the greater impact on morbidity and mortality. Catheter-related infections can affect the CVC exit site, the subcutaneous tunnel or, in the most severe cases, can induce bacteremia and sepsis (CRBSI, catheter-related blood stream infections). CRBSI prevention is of the utmost importance and can be achieved through educational activities for the dialysis personnel and through the use of specific protocols for CVC insertion and management. Indeed, it has been demonstrated that these measures allow a significant reduction of the number of CRBSI, to less than 1 episode per 1,000 catheter days.
\end{abstract}

Key words: Catheter-related blood stream infections (CRBSI), Protocol, CVC related complications, Hemodialysis

Conflict of interest: None.

Financial support: The preparation of this article was part of a project supported by a grant from the Italian Kidney Foundation, 2012.

Ricevuto: 19 Luglio 2013; Accettato: 24 Luglio 2013

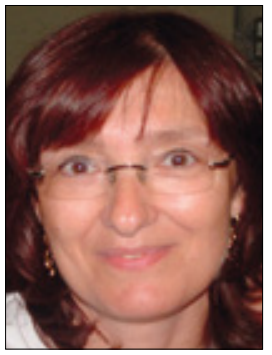

Marina Cornacchiari

\section{Introduzione}

Nonostante il numero elevato di ambulatori dedicati alla cura del paziente nella fase pre-dialitica, è esperienza relativamente frequente dover ricorrere, per necessità, al posizionamento di un catetere venoso centrale (CVC), temporaneo o permanente, per iniziare il trattamento dialitico. A conferma di questa osservazione, lo studio DOPPS $(1,2)$ ha evidenziato, sia nei pazienti incidenti che in quelli prevalenti, un trend in costante aumento nell'utilizzo di tali dispositivi. Nel periodo 2006-2010, l'utilizzo dei CVC tunnellizzati nei pazienti prevalenti in Italia è aumentato dall' $11 \%$ al $23.8 \%$, in Francia dal $14.3 \%$ al $16.8 \%$ e in Spagna dal $19 \%$ al $30 \%$, mentre, negli USA e in Canada, è passato rispettivamente dal $27.3 \%$ al $28.5 \%$ e dal $39 \%$ al $52 \%$. Solo il Giappone si mantiene a livelli molto bassi, pur registrando, nello stesso periodo, un aumento dallo $0.5 \%$ all'1.6\% (Fig. 1). Si è osservato come l'incremento nell'uso dei CVC non sia da correlarsi solamente all'aumento dell'età anagrafica e/o delle comorbilità nella popolazione afferente agli ambulatori nefrologici, in quanto il loro utilizzo è in netto aumento anche in pazienti giovani e non diabetici (3).

L'uso dei CVC è gravato da una dimostrata maggiore mortalità globale e cardiovascolare (4-9). È noto ormai da tempo che il CVC induce una risposta infiammatoria cronica che, a sua volta, può essere uno stimolo ai processi di aterogenesi $(10,11)$ e, dall'analisi multi-variata di diversi studi, è emersa una maggiore mortalità dei pazienti portatori di CVC, indipendentemente da altre condizioni, quali età, sesso, abitudini di vita, fumo e patologie associate (5-8). In particolare, uno studio eseguito in Olanda su 1000 pazienti dializzati (9) ha evidenziato come i pazienti portatori di CVC e di età maggiore di 65 anni abbiano un rischio di mortalità aumentato del 54\%, se confrontati con pazienti della stessa età portatori di FAV. Sempre nello stesso studio è stato osservato come $\mathrm{i}$ pazienti con età maggiore di 75 e portatori di CVC abbiano un rischio di mortalità aumentato dell' $83 \%$, se confrontati con pazienti della stessa età portatori di FAV come accesso vascolare per dialisi (9). 


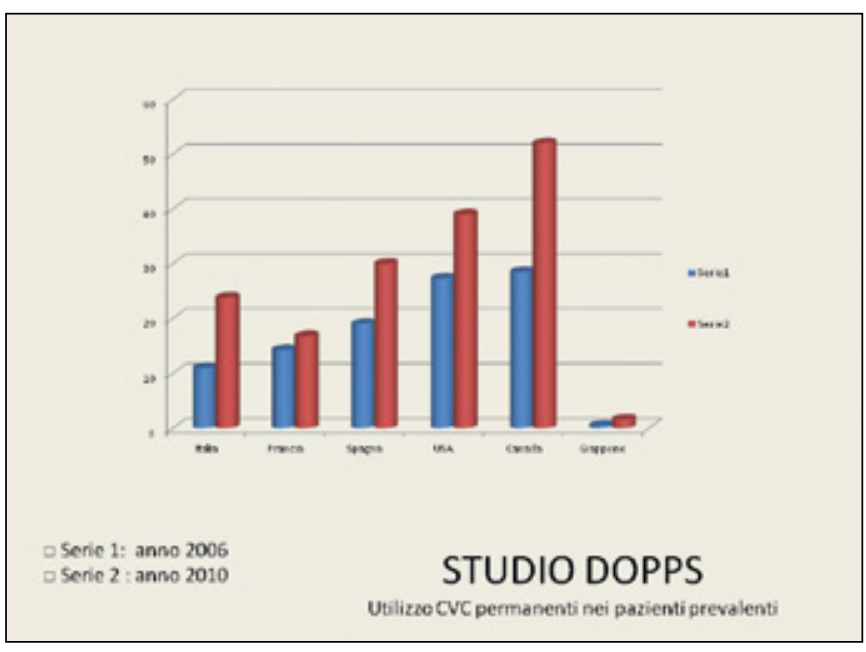

Fig. 1

\section{Complicanze legate ai CVC}

Le complicanze dei CVC possono dipendere dalla manovra del loro posizionamento e dalle conseguenze della permanenza del CVC nella sede in cui è stato inserito (12).

Tra le possibili complicanze legate alla manovra di posizionamento sono stati segnalati: la lacerazione della vena (in rari casi anche la perforazione della vena cava superiore o del cuore destro), la lesione dell'arteria con formazione di uno pseudoaneurisma e/o di una fistola arteriovenosa, la lesione di nervi contigui con paresi secondaria, lo pneumotorace, la lesione del dotto toracico, la perforazione della trachea e /o della laringe e le aritmie. Utilizzando una tecnica ecoguidata, il numero delle complicanze da inserimento del CVC si è ridotto moltissimo e, in alcune casistiche, si è quasi azzerato. Anche lo sviluppo tecnologico dei cateteri, ora meno rigidi rispetto al passato, ha contribuito a una riduzione degli eventi avversi. Quando si verificano, alcune di queste complicanze possono manifestarsi con sanguinamenti abbondanti, che possono, però, essere inapparenti quando si riversano verso spazi interni (come nel caso di un emotorace o di un emomediastino). Più frequentemente, un sanguinamento dall'emergenza di un CVC, specialmente nel caso dei CVC tunnellizzati, è determinato dall'uso eccessivo di un anticoagulante durante la procedura di inserimento o alla chiusura del catetere.

Tra le possibili complicanze dovute alla permanenza del CVC nella sede in cui è stato inserito sono state segnalate: infezioni, trombosi, dislocazione, rottura e difficoltà nella rimozione del CVC $(13,14)$.

Le infezioni sono una complicanza particolarmente rilevante dei CVC e la maggiore incidenza delle infezioni determina una maggiore mortalità per cause infettive $(15,16)$. L'uso di CVC all'inizio del trattamento dialitico si associa a un aumento del $47 \%$ delle ospedalizzazioni conseguenti alle infezioni (17).

Infine, i CVC possano rappresentare un problema per l'erogazione di un'adeguata dose dialitica $(18,19)$. Nonostante ciò,

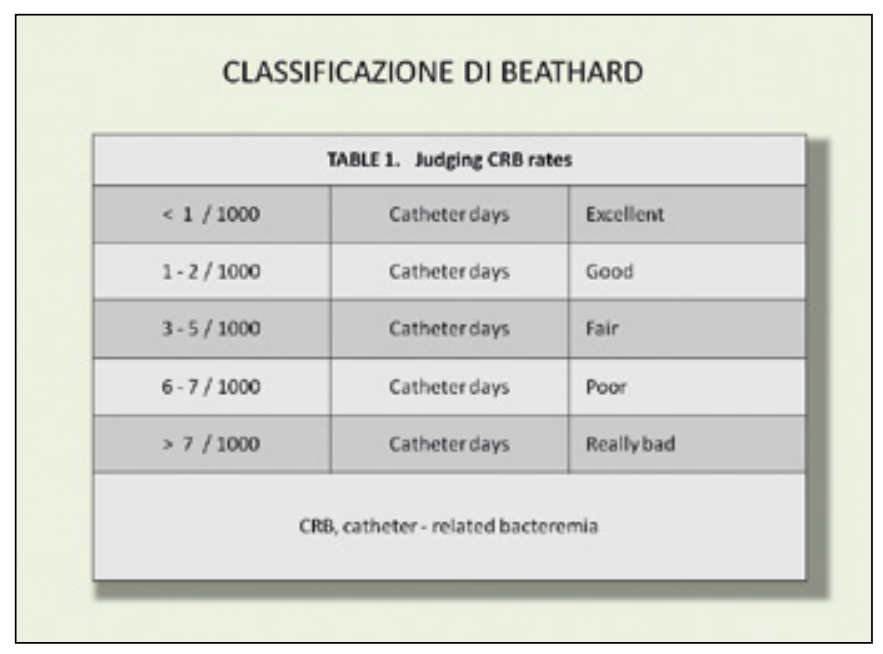

Fig. 2

come già ricordato, il loro uso è in costante aumento nella comunità nefrologica internazionale $(1,2,20)$.

\section{Infezioni ematiche correlate ai cateteri intra- vascolari (CRBSI, catheter related blood-stream infections)}

Le due maggiori complicanze che si incontrano con l'utilizzo dei CVC sono il malfunzionamento per ostruzione da trombosi (o anche, più raramente, per dislocamento o piegatura) $\mathrm{e}$ le infezioni. Queste due importanti complicanze sono spesso correlate, perché, in genere, un CVC malfunzionante necessita di frequenti disconnessioni per lavaggi, inversione delle linee e altre possibili manovre disostruenti, il che aumenta in modo esponenziale il rischio di contaminazione del CVC. L'infezione può essere extra-luminale, interessando l'area di emergenza (exit site) del CVC e/o il tunnel, oppure intra-luminale, con contaminazione diretta del lume del CVC, da dove è verosimile la diffusione di un'infezione al paziente.

La morbilità e la mortalità di cui sono gravate le CRBSI impongono l'adozione di misure per ottenere la loro massima prevenzione. Una prevenzione efficace consente, inoltre, un risparmio economico per la riduzione del numero di procedure e ricoveri, una riduzione dei danni al patrimonio vascolare del paziente e una riduzione del carico di lavoro per lo staff medico-infermieristico $(4,21,22)$.

L'incidenza delle infezioni CVC correlate (CRBSI) varia a seconda del tipo di CVC: da 3.8 a 6.6 episodi/1000 giorni di permanenza nei CVC temporanei a 1.1-5.5 episodi/1000 giorni di permanenza nei CVC tunnellizzati. Recentemente, Beathard (15), in base all'incidenza delle batteriemie riscontrate in 1000 giornate di permanenza, ha costruito una tabella che esprime 5 gradi di efficienza con estremi definiti really bad ( $>7$ infezioni/1000 giorni catetere) ed excellent $(<1$ infezioni/1000 giorni catetere) (Fig. 2). Tali valori devono rappresentare il termine di confronto di ogni centro dialisi, dove la maggiore incidenza di CRBSI indica un insufficiente e inefficiente protocollo di 
gestione del CVC e, viceversa, l'incidenza inferiore a 1 episodio di infezione/1000 giorni catetere rappresenta l'obiettivo da perseguire.

Le vie di propagazione delle CRBSI possono essere le seguenti: 1) contaminazione diretta del CVC durante il posizionamento del dispositivo o nelle manovre di apertura e chiusura, che sono ritenute responsabili dell' $80 \%$ circa delle CRBSI, 2) migrazione di micro-organismi dal sito di emergenza (soprattutto con i CVC temporanei), 3) diffusione ematogena da un focolaio infettivo localizzato in un'altra sede (rara) e 4) contaminazione di soluzioni somministrate per via endovenosa (rara).

È, quindi, importante sottolineare come la maggior parte delle infezioni non sia causata principalmente dalla "noncuranza" o dalle cattive condizioni igieniche del paziente, ma da manovre non corrette del personale medico-infermieristico durante l'assistenza in corso di dialisi. Pertanto se, in un centro dialisi, l'incidenza delle infezioni è elevata, è anche possibile intervenire sulle procedure assistenziali per correggere la situazione. Nei reparti di terapia intensiva, dove la permanenza dei pazienti e dei cateteri è relativamente breve, attualmente ci si pone l'obiettivo "zero infezioni", almeno nei pazienti con permanenza dei CVC tra 1 e 9 giorni (23). Nei centri dialisi, questo obiettivo sembra, al momento, impossibile da raggiungere, ma non è sbagliato porselo, per avvicinarsi il più possibile e rendere più sicuro l'utilizzo del CVC, uno strumento prezioso nei casi in cui un accesso vascolare arteriovenoso sia impossibile da ottenere o in cui possa essere mantenuto solo con interventi chirurgici e procedure interventistiche ripetute frequentemente. Lo staff medico-infermieristico è al centro della scena e di fronte a una grande responsabilità, potendo essere, in alcuni casi, il veicolo dell'infezione e delle sue conseguenze potenzialmente molto gravi. Dando per scontato che tutti gli operatori conoscano i concetti fondamentali per la prevenzione della trasmissione delle infezioni in ambiente ospedaliero, verosimilmente, nei centri in cui il numero delle infezioni è superiore ai livelli adeguati, nessun operatore è consapevole di quali siano i propri comportamenti a rischio. In genere si tratta, infatti, di un problema di scelta e, soprattutto, di applicazione delle corrette procedure, il che sposta la potenziale responsabilità dell'infezione sul team più che sul singolo individuo, tranne nei casi di palese violazione delle regole stabilite e condivise all'interno dello staff. Per tutto quanto sin qui espresso, deve essere chiaro che è proprio all'interno dello staff che devono essere ricercate, capite e risolte le cause delle infezioni, perché solo cambiando o modificando i nostri comportamenti possiamo fare una vera prevenzione e ridurre l'incidenza delle CRBSI.

\section{Prevenzione delle CRBSI}

La prevenzione inizia nel momento del posizionamento del CVC sia temporaneo che permanente. Risultano, in tal senso, importanti il lavaggio pre-chirurgico delle mani, l'utilizzo di mascherina e cuffia (per tutti gli operatori coinvolti nella procedura), l'uso di guanti e camici sterili e la conoscenza degli antisettici e dei loro tempi e modalità e tempi d'azione.

Il lavaggio delle mani serve a eliminare la flora batterica che colonizza gli strati superficiali della cute, in cui risiedono an- che gli organismi più spesso associati alle infezioni ospedaliere (quali pseudomonas e stafilococchi). Il lavaggio serve anche a ridurre la flora batterica residente, più resistente alla rimozione ma meno associata alle infezioni ospedaliere (24). L'uso di guanti sterili durante le procedure è obbligatorio. È importante tenere presente che i guanti non forniscono una protezione completa contro la contaminazione delle mani, in quanto anche un lavaggio accurato, assolutamente indispensabile, non elimina totalmente la flora batterica cutanea. Pertanto, l'uso dei guanti sterili non preceduto da un corretto lavaggio chirurgico delle mani non protegge completamente il paziente dai germi che le nostre mani possono veicolare. È altrettanto importante rispettare $\mathrm{i}$ tempi d'azione degli antisettici utilizzati sia durante il lavaggio delle mani che durante la preparazione del campo operatorio $(12,25)$.

La prevenzione continua durante le fasi di attacco e stacco della procedura dialitica. Questo è il momento più delicato nella gestione dei CVC perché può rappresentare una fonte di infezione dall'ambiente esterno se non viene utilizzata un'attenta procedura di prevenzione della contaminazione microbica. Esistono diversi protocolli in letteratura che si possono adottare, ma non sono ancora disponibili Linee Guida condivise sulle procedure da utilizzare e, in particolare, non vi è una chiara indicazione in merito alla necessità di eseguire l'attacco e lo stacco con due operatori (di cui uno sterile) o alla possibilità di procedere correttamente anche con un singolo operatore. Tra i protocolli di gestione del CVC disponibili c'è anche quello che da anni utilizziamo nel nostro centro e che descriviamo, con il quale è stato possibile ottenere, in base alla tabella di Beathard (15), il livello di eccellenza, costantemente da 10 anni, con un'incidenza di batteriemia inferiore a 1/1000 giornate di permanenza del CVC (26). Riteniamo fondamentale comprendere come non sia il protocollo in sé a produrre dei buoni risultati, ma l'aderenza stretta di tutto il personale medico e infermieristico al protocollo stesso.

Per quanto riguarda le procedure di allestimento dell'accesso vascolare, compreso l'inserimento dei CVC, nel nostro centro, da oltre 10 anni, sono stati individuati alcuni infermieri che affiancano un nefrologo dedicato all'aspetto chirurgico. È presente, quindi, un team dell'accesso vascolare che comprende uno staff dedicato alle procedure chirurgiche.

Gli infermieri aiutano attivamente il medico in posizionamento, sostituzione e rimozione dei CVC e supervisionano il lavoro dei colleghi nella gestione dei cateteri, controllando l'aderenza al protocollo in uso e monitorando l'incidenza di ogni tipo di infezione, così come di ogni problematica relativa al $\mathrm{CVC}$, che è subito condivisa con il nefrologo dedicato. Questo permette un'immediata presa in carico delle problematiche, così come la loro eventuale risoluzione.

Lo staff medico-infermieristico inoltre, si aggiorna periodicamente partecipando a corsi e/o a congressi e facendo degli AUDIT interni. Le informazioni vengono, in seconda battuta, condivise con il restante personale sanitario che opera nel servizio dialisi.

Ricordiamo che studi osservazionali suggeriscono come una percentuale troppo elevata di infermieri non specificamente addestrati alla gestione dei CVC e/o un elevato rapporto pazienti/infermieri si associa a un aumento dell'incidenza di 
CRBSI nelle unità di terapia intensiva (12).

Per finire, la prevenzione si completa con una corretta informazione al paziente, che deve essere parte attiva nella gestione del dispositivo e di tutte le problematiche ad esso correlate.

\section{Conclusioni}

L'uso sempre più frequente dei CVC nei pazienti dializzati comporta una crescente necessità di saper prevenire e gestire le complicanze ad essi correlate. Tra queste, le CRBSI sono quelle più temibili sia per l'enorme impatto su morbilità e mortalità che per gli alti costi a cui sono connesse.

La prevenzione è l'arma migliore a nostra disposizione per un adeguato controllo delle infezioni. Prevenire le CRBSI significa educare il personale sanitario in merito alle indicazioni.

\section{Riassunto}

L'uso dei cateteri venosi centrali (CVC) temporanei o permanenti è in costante aumento tra i pazienti emodializzati, ma questi dispositivi sono gravati da numerose complicanze, potenzialmente anche gravi. Tra queste, sicuramente le infezioni sono quelle con un maggiore impatto su morbilità e mortalità. Le infezioni correlate al CVC possono interessare il sito di emergenza del catetere e il tratto sottocutaneo dei cateteri tunnellizzati $\mathrm{o}$, nei casi più gravi, possono determinare batteriemie e/o setticemie (CRBSI, catheter related blood-stream infections). Importante è la loro prevenzione attraverso l'educazione del personale sanitario che gestisce tali dispositivi, associata a un buon protocollo di utilizzo degli stessi. Infatti, è dimostrato che, con un adeguato protocollo di inserimento e di gestione dei CVC, le CRBSI possono essere significativamente ridotte, al di sotto di 1 episodio ogni 1000 giorni-catetere.

Parole chiave: Infezioni CVC correlate (CRBSI), Protocollo, Complicanze da CVC, Emodialisi

Dichiarazione di conflitto di interessi: Gli Autori dichiarano di non avere conflitto di interessi.

Contributi economici agli Autori: La preparazione dell'articolo costituisce parte di un progetto sostenuto da un grant della Fondazione Italiana del Rene, anno 2012.

Indirizzo degli Autori:

Dr. ssa Marina Cornacchiari

Servizio di Nefrologia e Dialisi

Ospedale G. Fornaroli

Via Donatore di Sangue 51

20013 Magenta (MI)

marina.cornacchiari@ao-legnano.it

\section{Bibliografia}

1. 2010 Annual Report of the Dialysis Outcomes and Practice Patterns Study: Hemodialysis Data 1999-2008. Arbor Research Collaborative for Health, Ann Arbor, MI, USA. http://www. dopps.org/annualreport/html/mVAUType_c_TAB2010.htm. Visionato: 11/7/2013.

2. Rayner HC, Pisoni RL. The increasing use of hemodialysis catheters: evidence from the DOPPS on its significance and ways to reverse it. Semin Dial 2010; 23: 6-10.

3. Chaudhry M, Bhola C, Joarder M, et al. Seeing eye to eye: the key to reducing catheter use. J Vasc Access 2011; 12: 120-6.

4. Dhingra RK, Young EW, Hulbert-Shearon TE, Leavey SF, Port FK. Type of vascular access and mortality in U.S in hemodialysis patients Kidney Int 2001; 60: 1443-5.

5. Pastan S, Soucie M, McClennan WM. Vascular access and increased risk of death among hemodialysis patients. Kidney Int 2002; 62: 620-6.

6. Polkinghorne KR, McDonald SP, Atkins RC, Kerr PG. Vascular access and all-cause mortality: a propensity score analysis. J Am Soc Nephrol 2004; 15: 477-86.

7. Ravani P, Palmer SC, Oliver MJ, et al. Associations between hemodialysis access type and clinical outcomes: a systematic review. J Am Soc Nephrol 2013; 24: 465-77.

8. Bray BD, Boyd J, Daly C, et al. Vascular access type and risk of mortality in a national prospective cohort of haemodialysis patients. QJM 2012; 105: 1097-103.

9. Ocak G, Halbesma N, Le Cessie S, et al. Haemodialysis catheters increase mortality as compared to arteriovenous accesses especially in elderly patients. Nephrol Dial Transplant 2011;26: 2611-7.

10. Movilli E, Brunori G, Camerini C, et al. The kind of vascular access influences the baseline inflammatory status and epoietin response in chronic hemodialysis patients. Blood Purif 2006; 245: 387-93.

11. Hung AM, Ikizler TA. Hemodialysis central venous catheters as a source of inflammation and its implications. Semin Dial 2008; 21: 401-4.

12. Pittiruti M, La Greca A, Emoli A, Scoppettuolo G. Il protocollo ISALT 2 per l'impianto degli accessi venosi centrali: una proposta GAVeCeLT per un approccio più sicuro e costo efficace. Osp Ital Chir 2010; 359-68.

13. Lomonte $\mathrm{C}$, Basile C. Gestione del catetere venoso centrale: prevenzione della trombosi e della batteriemia. [Management of central venous catheter: prevention of thrombosis and bacteremia]. G Ital Nefrol 2009; 26 (1): 73-80.

14. Cornacchiari M, Zuccari M, Neri AL, et al. Due casi di difficile rimozione del catetere venoso centrale permanente. GTN\&D 2012; 24: 46-50.

15. Beathard GA, Urbanes A. Infection associated with tunneled he- 
modialysis catheters. Sem Dial 2008; 21: 528-38.

16. Oliver MJ, Rothwell DM, Fung K, Hux Je, Lok CE. Late creation of vascular access for hemodialysis and increased risk of sepsis. J Am Soc Nephrol 2004; 15: 1936-42.

17. Ng LJ, Chen F, Pisoni RL, et al. Hospitalization risks related to vascular access type among incident US hemodialysis patients. Nephrol Dial Transplant 2011; 26: 3659-66.

18. Cortez AJ, Paulson WD, Schwab SJ. Vascular access as a determinant of adequacy of dialysis. Semin Nephrol 2005; 25: 96-101.

19. Libardi S, Bacchini G, Pontoriero G. La gestione dell'accesso vascolare nei pazienti in dialisi: il contributo dello Studio DOPPS. TN\&D 2010; 22: 27-33.

20. Ethier J, Mendelssohn DC, Elder SJ, et al. Vascular access use and outcomes: an international perspective from the dialysis outcomes and practice patterns study. Nephrol Dial Transplant 2008; 23: 3219-26.

21. Xue JL, Dahl D, Ebben JP, Collins AJ. The association of initial hemodialysis access type with mortality outcomes in elderly Medicare ESRD patients. Am J Kidney Dis 2003; 42: 1013-9.
22. Xue JL, Dahl D, Ebben JP, Collins AJ. Vascular access and increased risk of death among hemodialysis patients. Kidney Int 2002; 62: 620-6.

23. Worth LJ, McLaws ML. Is it possible to achieve a target of zero central line associated bloodstream infections? Curr Opin Infect Dis 2012; 25: 650-7.

24. Azienda Ospedaliera Ospedale Niguarda Ca' Granda. Il lavaggio delle mani e l'uso dei guanti. Linee guida evidence based. Data elaborazione: aprile 2005. Data revisione: luglio 2007. Disponibile all'indirizzo internet: http:/www.ospedaleniguarda.it/ resources/Lavaggio_mani_797.pdf (visionato il 12/7/2013).

25. Boyce JM, Pittet D. Guideline for hand hygiene in healthcare setting. MMWR 2002; 51: RR-16. Disponibile all'indirizzo internet: http://www.cdc.gov/mmwr/preview/mmwrhtml/ rr5116a1.htm (visionato il 12/7/2013).

26. Cornacchiari M, Heidempergher M, Guastoni C, et al. Effectiveness of a protocol for the prevention of hemodialysis venous catheter-related infections. J Vasc Access 2011; 12: 313-7. 